\title{
Pharmacokinetics of naftopidil, a novel anti-hypertensive drug, in patients with hepatic dysfunction
}

\author{
M.J.G. Farthing, E.M. Alstead, S.M.L. Abrams' ${ }^{1}$, G. Haug 4 , A. Johnston', \\ R. Hermann ${ }^{2}$, G. Niebch ${ }^{3}$, P. Ruus ${ }^{2}$, K.H. Molz ${ }^{4}$ and P. Turner ${ }^{1}$
}

Department of Gastroenterology and ${ }^{1}$ Department of Clinical Pharmacology, St Bartholomew's Hospital, London ECIA $7 B E, U K,{ }^{2}$ Department of Clinical Pharmacology and ${ }^{3}$ Department of Biochemistry, ASTA Medica AG, Frankfurt/M and ${ }^{4} L A B$ GmbH, Neu-Ulm, Germany

Summary: The pharmacokinetics of naftopidil, a novel alpha-1 adrenoceptor-blocking antihypertensive, were investigated in ten patients $(9 \mathrm{M} / 1 \mathrm{~F})$ with hepatic dysfunction after oral administration (50 $\mathrm{mg}$, tablet) and after an intravenous infusion of $5.0 \mathrm{mg}$ over 2 minutes. Results were compared to a control group of 12 healthy subjects $(6 \mathrm{M} / 6 \mathrm{~F})$ of a previous investigation, which was carried out according to the identical study protocol.

The pharmacokinetic parameters obtained for the i.v. administration were comparable in both groups (half life 3.6 \pm 3.4 hours in liver-impaired subjects versus $3.3 \pm 2.1$ hours in controls; clearance $11.9 \pm 4.7 \mathrm{ml} / \mathrm{minute} / \mathrm{kg}$ versus $11.0 \pm 1.6 \mathrm{ml} / \mathrm{minute} / \mathrm{kg}$ ).

Following oral administration the plasma levels and half-life times of naftopidil were significantly increased in liver impairment $\left(t_{1} 16.6 \pm 19.3\right.$ hours versus $5.4 \pm 3.2$ hours in controls; $\left.P=0.012\right)$. Mean values for the absolute bioavailability in patients with hepatic dysfunction were significantly higher (mean $75 \%$, median $53 \%$, range $13.4-211.0 \%$ ) compared to healthy subjects (mean $17 \%$, median $16 \%$, range 6.7-29.6\%, $P=0.001)$.

Reduction of functional hepatic blood flow in chronic liver disease or, as evidenced in one case as a consequence of shunt surgery, is the probable cause of the observed alteration in naftopidil kinetics. This phenomenon occurred only following the oral $50 \mathrm{mg}$ dose whereas the intravenous $5 \mathrm{mg}$ dose obviously still could be normally handled. Naftopidil demethylation and hydroxylation were both less and non-uniformly affected.

The pharmacokinetic findings suggest that in patients with severe hepatic impairment or evidence for marked changes in hepatic blood flow the dose of naftopidil may require adjustment to the lower end of the therapeutic range and/or may be limited to once daily. However, before definite conclusions can be drawn, further steady-state studies are required. Despite the pharmacokinetic discrepancies no difference in drug tolerability was seen between patients and healthy subjects.

\section{Introduction}

Naftopidil is a novel phenylpiperazine vasodilator drug with selective alpha-1 adrenoceptor-blocking activity, which is undergoing clinical evaluation in patients with essential hypertension. ${ }^{1}$ Two active metabolites with similar potency to the parent compound are found in the blood and contribute to the therapeutic effects after oral administration.

In general, vasodilatation and the lowering of blood pressure by naftopidil are not accompanied by reflex tachycardia and cause no 'prazosin-like' first-dose phenomenon. ${ }^{2}$ Apart from a peripheral vasodilator effect based upon alpha-1 adrenoceptor blockade, an additional calcium antagonist action $^{3,4}$ as well as a centrally mediated reduction of peripheral sympathetic tone due to the interaction

Correspondence: A. Johnston, Ph.D.

Accepted: 29 October 1993 of naftopidil with central 5-hydroxytryptamine receptors, ${ }^{3}$ has been suggested. Pharmacokinetic studies in healthy volunteers, elderly and patients with renal impairment exhibited an extensive hepatic metabolism of naftopidil with a total plasma clearance of $9-11 \mathrm{ml} / \mathrm{minute} / \mathrm{kg}$, suggesting a close relationship to the liver plasma flow. Therefore, naftopidil pharmacokinetics have now been examined after single intravenous (i.v.) and oral administration to patients with hepatic dysfunction.

\section{Patients and methods}

\section{Study design}

Both studies (the recent study in subjects with hepatic impairment as well as the previous trial in 
healthy volunteers) were performed according to the same study protocol based on an open-label randomized two-period cross-over design. The clinical part of the investigation in liver-impaired subjects was performed in two centres - St Bartholomew's Hospital, London, UK and LAB GmbH, Neu-Ulm, Germany. All subjects were randomly assigned to receive on two occasions 8 days apart a single oral and intravenous administration of naftopidil. They were fasted from 22.00 hours the previous day, and were instructed to take no alcohol or drugs known to influence drug metabolism throughout the course of the study. Naftopidil was administered between 07.00 and 08.00 hours on an empty stomach. It was given either as an i.v. infusion within 2 minutes, or as a $50 \mathrm{mg}$ tablet with $150 \mathrm{ml}$ tap water. After test drug intake, all subjects remained in a supine position for 4 hours. Blood pressure by mercury sphygmomanometer and pulse rate were measured in a supine position on the right arm after subjects had been rested for at least 3 minutes. Heparinized blood samples were taken from an indwelling venous cannula before drug administration, at the end of the intravenous infusion, and at $5,10,15,30$, 45 minutes, and $1,1.5,2,3,4,6,8,10,12,16,20,24$, 36,48 and 60 hours post dose. Plasma samples were stored at $-20^{\circ} \mathrm{C}$ until analysed.

\section{Patients}

Ten patients $(9 \mathrm{M}, 1 \mathrm{~F})$ with hepatic impairment but with no other clinically significant disease were studied. They were $48-69$ years old (mean $58 \pm 6$ years) and their weights ranged from 55 to $92 \mathrm{~kg}$ (mean $74 \pm 11 \mathrm{~kg}$ ). Seven had post-alcoholic cirrhosis and one cirrhosis due to chronic active hepatitis. The other two patients suffered either from chronic active hepatitis, or fatty liver together with a history of portal hypertension due to portal vein thrombosis with subsequent palliative splenorenal shunt surgery. The severity of the liver disease was assessed according to the Child classification into Grades A, B or C. ${ }^{5,6}$ Five patients were treated with other drugs.

All six patients of the German centre were additionally subjected to comprehensive liver function tests including aminopryine breath test, an indirect measurement of decreased functional hepatic microsomal mass ${ }^{7,8}$ according to Pauwels et al. . Caffeine $^{10}$ and paracetamol acetaminophen clearance $^{11}$ were used to investigate the metabolic capacity of phase I and phase II pathway. In addition, fasting bile acids were determined as a parameter mainly reflecting porto-systemic shunting $^{12}$ and diminished hepatic clearance of bile acids. ${ }^{13}$

\section{Plasma assay}

Plasma naftopidil, desmethyl-naftopidil $2 \mathrm{HCl}$, and (phenyl)hydroxy-naftopidil $2 \mathrm{HCl}$ concentrations were measured after extraction by reversedphase high-performance liquid chromatography (HPLC) with fluorimetric detection according to a published internal standard method, ${ }^{14}$ starting with $0.5 \mathrm{ml}$ plasma aliquots and attaining a lower limit of quantification of $5 \mathrm{ng} / \mathrm{ml}$ for all three compounds. Daily calibration lines from control plasma spiked at $5,10,20,50,100,200 \mathrm{ng} / \mathrm{ml}$ served for the evaluation of the unknowns.

\section{Pharmacokinetic evaluation}

Pharmacokinetic analysis of the data was carried out using the STRIPE computer program. ${ }^{15}$

\section{Statistical analysis}

Although data are from different trials, explorative statistical tests were performed. Differences in pharmacokinetic measurements between the two groups of subjects were tested using two-sided rank tests (Mann-Whitney-Wilcoxon) for independent observations. $P<0.05$ was considered statistically significant in an explorative sense as no hypotheses have been formulated in advance.

The protocol and the performance of the study were in accordance with the ethical guidelines of the Helsinki Declaration. All subjects gave their written informed consent to take part, after the protocol had been approved by the Ethics Committees of the General Medical Council of Bavaria and the District Ethics Committee of St Bartholomew's Hospital, London.

\section{Results}

Patients and controls from a previous study of identical design ${ }^{16}$ were well matched regarding weight, height and laboratory parameters of renal function, ${ }^{*}$ but control subjects were younger than the patients (mean age $27 \pm 2.8$ versus $58.1 \pm 6.2$ ) and additionally the control group had a different sex distribution (patients $9 \mathrm{M} / 1 \mathrm{~F}$; healthy subjects $6 \mathrm{M} / 6 \mathrm{~F}$ ). In both cases, experimental data were evaluated compartment-free with a oneexponential terminal decay. In control subjects, for 18 hours onwards, concentrations of naftopidil were often below the lower limit of quantification. The metabolites desmethyl-naftopidil and (phenyl) hydroxy-naftopidil were not detectable after intravenous administration, in patients or in healthy

*For interested readers, detailed case data may be available on request. 
subjects. After oral administration measurable plasma concentrations of these metabolites were found in all healthy subjects but only in some of the patients. Concentration-time curves are plotted in Figures 1 and 2, respectively.

The pharmacokinetic parameters obtained for the i.v.-administration were comparable in both groups (plasma half-life $3.6 \pm 3.4$ hours in liverimpaired patients versus $3.3 \pm 2.1$ hours in controls; plasma clearance $11.9 \pm 4.7 \mathrm{ml} / \mathrm{minute} / \mathrm{kg}$ versus $11.0 \pm 1.6 \mathrm{ml} /$ minute $/ \mathrm{kg}$ ), except the maximum plasma concentration $\left(C_{\max }\right)$.

Following oral administration the plasma levels and half-life times of naftopidil were significantly increased in liver impairment $\left(t_{1 / 2} 16.6 \pm 19.3\right.$ hours versus $5.4 \pm 3.2$ hours in controls; $P=0.012$ ). Mean values for the absolute bioavailability in

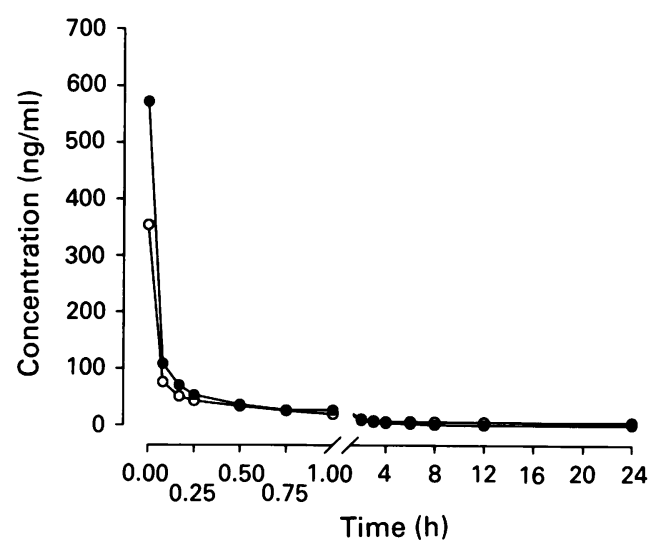

Figure 1 Mean plasma level-time courses of naftopidil after i.v. administration of $5 \mathrm{mg}$. $\mathrm{O}=10$ patients with hepatic impairment; $=12$ healthy young volunteers.

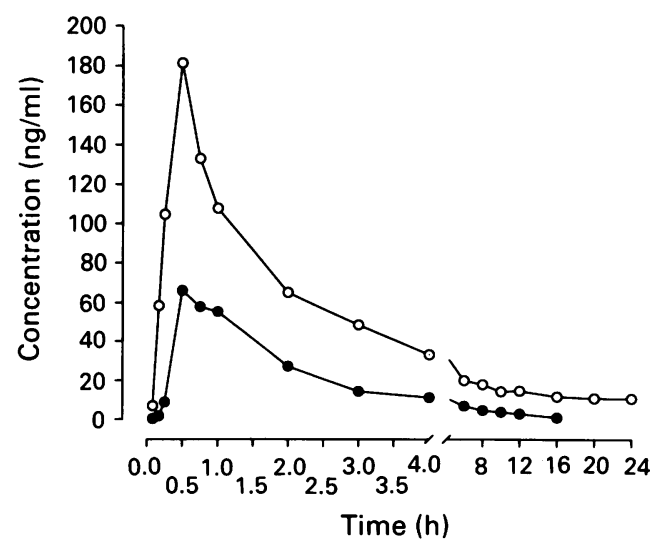

Figure 2 Mean plasma level-time courses of naftopidil after oral administration of $50 \mathrm{mg}$ (tablet). $\mathrm{O}=10$ patients with hepatic dysfunction; $=12$ healthy young volunteers. patients with hepatic dysfunction were significantly higher (mean $75 \%$, median $53 \%$, range $13.4-$ $211.0 \%$ ) compared to healthy subjects (mean $17 \%$, median $16 \%$, range $6.7-29.6 \%, P=0.001)$. In contrast to the i.v. measurements, peak concentrations after oral administration were considerably higher in patients than in controls (Figure 2).

Considering the results of the liver function tests, there was no correlation between the disposition of naftopidil and the results of aminopyrine breath test, caffeine- or paracetamol-clearances, respectively. In contrast a significant decrease in functional hepatic blood flow as evidenced by elevated serum bile acids, was related to a marked increase in naftopidil 'area under the curve' (Figure 3). In addition, the patient in whom a spleno-renal shunt had been fashioned, also exhibited a considerably impairment in his ability to handle naftopidil, comparable to those patients with the largest values for fasted serum bile acids.

Despite the observed significant impact of chronic hepatic disease on naftopidil kinetics, the drug was generally well tolerated by the patients. Orthostatic hypotension or tachycardia were not detected in any case. Physical examination within 8 days of completion of the study showed no pathological findings which could be referred to naftopidil, and no significant changes in laboratory tests of haematological, renal or hepatic function were detected.

\section{Discussion}

The analytical performance of the plasma naftopidil assay used in this study was similar to that

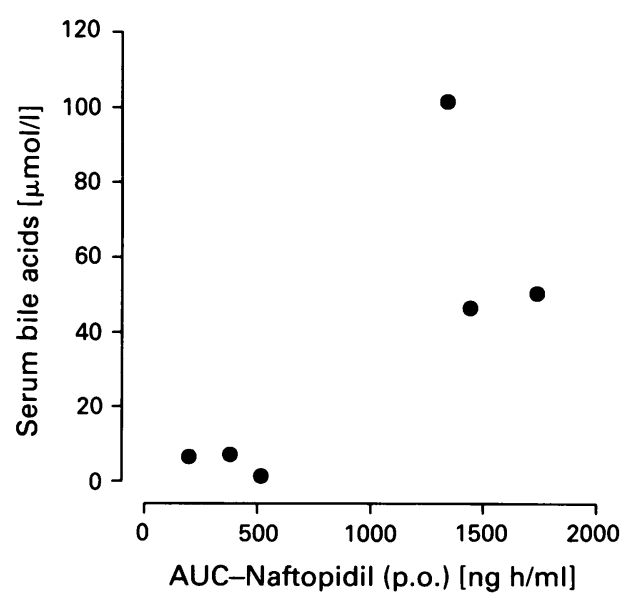

Figure 3 Fasted serum bile acid concentration, as a parameter of functional hepatic flow, plotted against the area under the curve of naftopidil in six patients with liver disease. 
used in previous investigations. ${ }^{16}$ Thus, it is permissible to compare the results obtained in these different pharmacokinetic studies. However, it should be noted that the patients with liver impairment were older than the controls. Although some impairment of hepatic drug metabolism may occur on ageing, the pharmacokinetics of naftopidil in the elderly were shown to agree well with those in young healthy volunteers (unpublished results). In the latter population, the absolute bioavailability of orally administered naftopidil ( $50 \mathrm{mg}$ tablet) has been determined to be about $17 \% .^{15}$ The mechanism responsible for this low value is presumed to be an extensive first-pass metabolism, since nearly complete ${ }^{14} \mathrm{C}$-absorption could be seen following oral $\left[{ }^{14} \mathrm{C}\right]$ naftopidil administration (unpublished results). Therefore, naftopidil is obviously a representative of compounds with a high extraction ratio. In general, the influence of liver disease on the clearance of highly extracted drugs is determined predominantly by changes in hepatic blood flow, rather than by other parameters of hepatic drug elimination such as intrinsic hepatic clearance and protein binding. ${ }^{17}$ Because hepatic disease often results in reduced hepatic blood flow, sometimes with extensive portosystemic shunting, the clearance of highly extracted drugs is most consistently reduced in this clinical setting. One major effect of portosystemic shunting is a substantial reduction in first-pass metabolism $^{6}$ which may considerably impair the ability of patients to handle such drugs without them necessarily displaying other marked features of liver disease. ${ }^{18}$ This particular clinical constellation was present in one of our patients, with spleno-renal shunt surgery following portalvenous thrombosis without cirrhosis.

In conclusion, our data indicate an impairment of the disposition of naftopidil in patients with chronic liver disease after an oral $50 \mathrm{mg}$ dose and are consistent with high clearance drugs. There was no pharmacokinetic difference following the i.v. administration of $5 \mathrm{mg}$. Whereas pharmacokinetics after oral dosing with 25,50 and $100 \mathrm{mg}$ of naftopidil were independent of dose in healthy subjects (unpublished results), the present findings suggest that non-linear pharmacokinetics start at considerable lower doses in patients with reduction in functional hepatic flow in liver disease. However, it is possible that the chosen $5 \mathrm{mg}$ i.v. dose was too low to reflect drug metabolism, rather than distribution.

The pharmacokinetic findings suggest that in patients with severe hepatic impairment or evidence for marked changes in hepatic blood flow the dose of naftopidil may require adjustment to the lower end of the therapeutic range and/or may be limited to once daily. Before definite conclusions can be drawn, further steady-state studies are required. Despite the pharmacokinetic discrepancies, no difference in drug tolerability was seen between patients and healthy subject.

\section{References}

1. Anon. Naftopidil. Drugs Future 1987, 12: 31-33.

2. de Mey, C., Roll, S., Erb, K., Köhnlein, A., Hermann, R. \& Belz, G.G. Differential effects of naftopidil and prazosin on the hemodynamic responses to passive upright tilt in healthy normotensives. Naunyn-Schmiedebergs Arch Pharmacol 1992, 346: C43.

3. Borbe, H.O., Untch, H. \& Sponer, G. Naftopidil: receptor binding profile of a novel vasodilator. NaunynSchmiedeberg's Arch Pharmacol 1989, 340: R62.

4. Borbe, H.O. \& Peter, G. Radioreceptor assay (RRA) for the determination of al-adrenoceptor-binding material in rat plasma following single oral administration of naftopidil (50 mg/kg). Arzneim Forsch 1990, 40: 253-256.

5. Child, C.G. The Liver and Portal Hypertension. Saunders, Philadelphia, 1964, p. 50.

6. Howden, C.W., Birnie, G.G. \& Brodie, M.J. Drug metabolism in liver disease. Pharm Ther 1989, 40: 439-474.

7. Hepner, G.W. \& Vesell, E.S. Assessment of aminopyrine metabolism in man after oral administration of 14Caminopyrine. Effects of phenobarbital, disulfiram, and portal cirrhosis. N Engl J Med 1974, 291: 1384-1388.

8. Bircher, J., Kupfer, A. \& Gikalov, I. Aminopyrine demethylation measured by breath analysis in cirrhosis. Clin Pharmacol Ther 1976, 20: 484-492.

9.. Pauwels, S. Geubel, A.P., Dive, C. \& Beckers, C. Breath ${ }^{14} \mathrm{CO}_{2}$ after intravenous administration of $\left[{ }^{14} \mathrm{C}\right]$ aminopyrine in liver diseases. Dig Dis Sci 1982, 27: 49-56.

10. Wahlländer, A., Mohr, S. \& Baumgartner, G. Assessment of hepatic function: comparison of caffeine clearance in serum and saliva at day and at night. $J$ Hepatol 1990, 10: 129-137.
11. Rawlins, M.D., Henderson, D.B. \& Hijab, A.R. Pharmacokinetics of paracetamol (acetaminophen) after intravenous and oral administration. Eur $J$ Clin Pharmacol 1977, 11: 283-286.

12. Ohkubo, H., Okuda, K., Iida, S. et al. Role of portal and splenic vein shunts and impaired hepatic extraction in the elevated serum bile acids in live cirrhosis. Gastroenterology 1983, 86: 514-520.

13. Gilmore, I.T. \& Thompson, R.P.H. Plasma clearance of oral and intravenous cholic acid in subjects with and without chronic liver disease. Gut 1980, 21: 123-127.

14. Niebch, G., Borbe, H.O. \& Besenfelder, E. High-performance liquid chromatography of naftopidil, a novel antihypertensive drug, and two metabolites in human plasma. $J$ Chromatogr 1990, 534: 247-252.

15. Johnston, A. \& Woollard, R.C. STRIPE: an interactive computer programme for the analysis of drug pharmacokinetics. J Pharmacol Meth 1983, 9: 193-199.

16. Birck, K.M., Riethmüller-Winzen, H., Borbe, H.O. \& Niebch, G. Naftopidil: tolerability, pharmacokinetics and bioavailability following single doses of naftopidil intravenous injection, tablet, capsule, and solution in healthy volunteers. Naunyn-Schmiedeberg's Arch Pharmacol 1990, 342: R4.

17. Bass, N.M. \& Williams, R.L. Guide to drug dosage in hepatic disease. Clin Pharmacokinet 1988, 15: 396-420.

18. Forrest, J.A.H., Finlayson, N.D.C., Adjepon-Yamoah, K.K. \& Prestcott, L.F. Antipryine, paracetamol and lignocaine elimination in chronic liver disease. $\mathrm{Br}$ Med $J$ 1977, 1: 1384-1387. 